\title{
High-dose-rate interstitial brachytherapy with hypoxic radiosensitizer KORTUC II for unresectable pelvic sidewall recurrence of uterine cervical cancer: a case report
}

\author{
Mio Nakata, MD', Ken Yoshida, MD², Taiju Shimbo, MD', Nobuhiko Yoshikawa, MD', Hiroto Yoshioka, MD', \\ Akihiro Hori, MD!, Chikara Sato, MD!, Yasuo Uesugi, MD³, Yuhei Kogata, MD², Koji Masui, MD ${ }^{5}$, Naoya Murakami, MD ${ }^{6}$ \\ Tairo Kashihara, MD6. Hironori Akiyama, DDS7, Nikolaos Tselis, MD², Masahide Ohmichi, MD4, Keiji Nihei, MD' \\ 'Department of Radiation Oncology, Osaka Medical College, Japan, ²Department of Radiology, Kansai Medical University Medical Center, \\ Japan, ${ }^{3}$ Department of Rehabilitation Sciences, Faculty of Allied Health Sciences, Kansai University of Welfare Sciences, Japan, ${ }^{4}$ Department \\ of Obstetrics and Gynecology, Osaka Medical College, Japan, ${ }^{5}$ Department of Radiology, Kyoto Prefectural University of Medicine, Japan, \\ ${ }^{6}$ Department of Radiation Oncology, National Cancer Center Hospital, Japan, ${ }^{7}$ Department of Oral Radiology. Osaka Dental University \\ Japan, ${ }^{8}$ Department of Radiation Oncology, Johann Wolfgang Goethe University Frankfurt, Germany
}

\begin{abstract}
In order to improve oncologic outcomes in radiotherapy treatments of patients with unresectable pelvic sidewall recurrences of uterine cervical cancer, we combined high-dose-rate interstitial brachytherapy (HDR-ISBT) with newly tested hypoxic radiosensitizer Kochi oxydol-radiation therapy for unresectable carcinomas (KORTUC II), an enzyme-targeting radiosensitization treatment involving intra-tumoral injection of sodium hyaluronate mixed with hydrogen peroxide. We report on a 63-year-old patient referred to our department with an extensive pelvic sidewall recurrence of uterine cervical cancer after initial hysterectomy. The tumor size was $55 \times 25 \times 80 \mathrm{~mm}$, with a calculated volume of $89.7 \mathrm{cc}$. Whole pelvic irradiation of $50 \mathrm{~Gy}$ in 25 fractions was administered, combined with weekly cisplatin injections. KORTUC II injections were given two times: at day 21 (42 Gy) and at day 24 (48 Gy). After finishing whole pelvic irradiation, HDR-ISBT of 25 Gy in 5 fractions b.i.d. over 3 days was administered. KORTUC II was also injected at the time of implantation. Dose-volume histogram $(\mathrm{DVH})$ values for clinical target volume were $\mathrm{D}_{90}, \mathrm{D}_{98}$, and $\mathrm{D}_{100}$ of 6.0, 5.0, and 3.5 Gy per fraction, respectively. $\mathrm{D}_{2 \mathrm{cc}}$ values were 2.1, 4.1, 3.2, and 2.0 Gy per fraction for the bladder, rectum, sigmoid colon, and small bowel, respectively. No acute adverse events $\geq$ grade 3 were observed. Repeated grade 3 pyelonephritis occurred as a late complication at 11,24, and 26 months after the treatment, and was successfully resolved with antibiotics. Moreover, grade 2 late toxicity was documented, including sciatic neuralgia, lower limb lymphedema, and urinary incontinence. At present, 32 months after HDR-ISBT, the patient remains free of disease, with no toxicity-related deterioration in physical condition.

Key words: pelvic sidewall recurrence, uterine cervical cancer, high-dose-rate, interstitial brachytherapy, KORTUC.

\section{Purpose}

Radical radiotherapy treatment for pelvic recurrence of uterine cervical cancer poses a clinical challenge due to proximity of organs at risk (OARs) to the tumor site. In particular, pelvic sidewall recurrences carry significant morbidity risk, prohibiting aggressive treatment due to involvement of large vessels and nerves. In such cases, an implementation of curative surgery is also limited [1], and treatment outcomes of external beam radiotherapy (EBRT) with or without chemotherapy, are similarly unsatisfactory [2]. Interstitial brachytherapy (ISBT) may provide an effective alternative for this poor-prognosis population. ISBT combines a lesion-specific approach of surgery with a tumoricidal capability of radical radiotherapy by escalating biologically effective dose to the treatment target, while ameliorating conformity. Versatility of intra-target dose modulation inherent to brachytherapy
Address for correspondence: Ken Yoshida, MD, Department of Radiology, Kansai Medical University Medical Center, 10-15, Fumizono-cho, Moriguchi, Osaka 570-8507, Japan, phone: +81-6-6992-1001, fax: +81-6-6992-4846, e-mail: yoshidaisbt@gmail.com
Received: 16.05 .2020

Accepted: 16.09 .2020

Published: 16.12 .2020 
offers greater control and ability to directly deliver higher doses to the total tumor, while selectively reducing the dose to OARs. Furthermore, technical advances, such as image-guided implantation, template-based approaches, and anatomy-oriented treatment planning have shown significant improvements in the treatment of recurrent uterine cervical cancer with low-dose-rate (LDR), pulsed-dose-rate (PDR), and high-dose-rate (HDR)-ISBT $[3,4,5,6,7,8,9,10,11,12,13,14,15]$. Our own experience with three-dimensional (3D) image-guided HDR-ISBT for recurrent uterine cervical and endometrial cancers after primary surgery is reflected in the published results of 56 patients with 3-year local control of $75 \%[16,17]$.

In spite of the enormous potential of HDR-ISBT, various factors, including complex lesion shapes and pubic arch interference may impair implantation and adequate irradiation, especially in pelvic sidewall recurrences. In order to improve the outcomes of such patients, we implemented a new hypoxic radiosensitizer in the radiotherapy of unresectable carcinomas (Kochi oxydol-radiation therapy for unresectable carcinomas - KORTUC). Ogawa et al. demonstrated that radioresistance of the human osteosarcoma cell line HS-Os-1 arises from low levels of reactive oxygen species (ROS) formation following irradiation, which in turn may result from a strong free radical scavenging ability of the cells, particularly scavenging of hydroxyl radicals. Therefore, they examined the effects of various doses of irradiation in the presence of $0.1 \mathrm{mM}$ hydrogen peroxide in a culture medium. They found that irradiation with 10 or $20 \mathrm{~Gy}$, in the presence of $0.1 \mathrm{mM}$ hydrogen peroxide, induced ROS formation, oxidative DNA damage, dysfunction of the mitochondrial membrane potential, and early apoptotic changes in the human osteosarcoma cell line HS-Os-1. Interestingly, ROS formation and oxidative DNA damage were scarcely seen in a response to irradiation of up to $30 \mathrm{~Gy}$, as shown in their previous study. The authors concluded that the modality of irradiation combined with a low concentration of hydrogen peroxide $(0.1 \mathrm{mM})$ may be found useful in clinical radiotherapy [18]. Based on these results, they clinically evaluated the effectiveness of this enzyme-targeting radiosensitization treatment, using a superficial coating of hydrogen peroxide (the KORTUC I method) and intra-tumoral injections of sodium hyaluronate mixed with hydrogen peroxide (the KORTUC II method) $[19,20]$. However, in comparison to EBRT, there are limited data on a hypoxic radiosensitization approach with ISBT. In this report, we describe a case of pelvic sidewall recurrence treated with HDR-ISBT combined with KORTUC II.

\section{Clinical case}

A 63-year-old female patient was referred to our department with a pelvic sidewall recurrence of uterine cervical cancer. Initially, the patient was treated with two cycles of neoadjuvant chemotherapy (intra-arterial cisplatin infusion and intravenous irinotecan). Irinotecan $70 \mathrm{mg} / \mathrm{m}^{2}$ on day 1 and 8 , and cisplatin $75 \mathrm{mg} / \mathrm{m}^{2}$ on day 2 were administered as one cycle. Subsequently, she underwent a radical hysterectomy (ypT2a2 ypN1 cM0 pR0, squamous cell carcinoma), followed by six cycles of adjuvant chemotherapy consisting of paclitaxel plus carboplatin. Paclitaxel at $175 \mathrm{mg} / \mathrm{m}^{2}$ and carboplatin at an area under the curve of 5 were administered as one cycle. Twenty-three months after the surgery, the patient developed an unresectable, left-sided pelvic sidewall recurrence. Her clinical symptomatology consisted of perianal pain. Magnetic resonance imaging (MRI) revealed a large lesion of $55 \times 25 \times 80 \mathrm{~mm}$ (Figure 1A), with an associated pathologic uptake of 18F-2-deoxy-glucose (FDG) in positron emission tomography (PET)/computed tomography (CT), without distant metastases (Figure 1B). Tumor marker (SCC) was elevated to 6.7 (normal range, < 1.5). Even though a biopsy was not performed, the lesion was clinically diagnosed as a recurrence.

After interdisciplinary assessment, followed by informed consent, the patient was offered EBRT to the whole pelvis up to 50 Gy in 25 fractions, with weekly intravenous cisplatin $\left(40 \mathrm{mg} / \mathrm{m}^{2}\right)$ injections. We injected KORTUC II in total three times, with two times performed
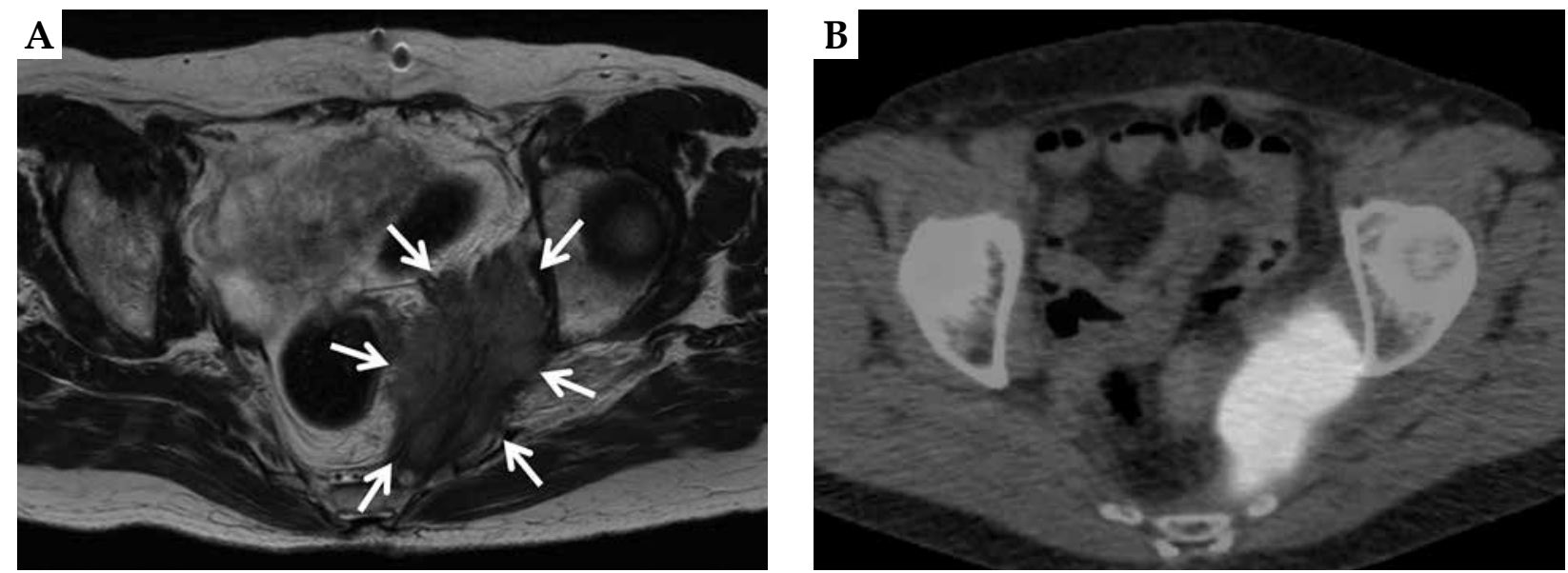

Fig. 1. A) Magnetic resonance imaging depicting the left-sided uterine cervical cancer recurrence. In the T2-weighted sequence, the large tumor $(55 \times 25 \times 80 \mathrm{~mm})$ at the left pelvic sidewall is clearly demarcated (white arrows). B) Positron emission tomography/computed tomography scan image of the same patient. A pathologic uptake of 18F-2-deoxy-glucose (FDG) is shown in correspondence to the MRI findings 
in EBRT and final injection during ISBT. KORTUC II was additionally injected into the tumor lesion at day 21 (dose point, $42 \mathrm{~Gy}$ ) and day 24 (dose point, $48 \mathrm{~Gy}$ ) during the EBRT. In our hospital, $0.5 \mathrm{ml}$ of $3 \%$ hydrogen peroxide, $2.5 \mathrm{ml}$ of sodium hyaluronate (Adant Dispo intra-articular injection $\left.{ }^{\circledR}\right)$, and $1 \mathrm{ml}$ of $1 \%$ lidocaine $\left(X_{\text {ylocaine }}{ }^{\circledR}\right)$ were mixed in a single syringe to formulate KORTUC II, and 1 to 3 syringes $(4-12 \mathrm{ml})$ were injected depending on the tumor size. If the tumor diameter was less than $30 \mathrm{~mm}$, only one syringe $(4 \mathrm{ml})$ of KORTUC II was injected at each time point. If the tumor diameter was $30-60 \mathrm{~mm}$, 2 syringes $(8 \mathrm{ml})$ of KORTUC II were injected at each time point, and if the tumor diameter was more than $60 \mathrm{~mm}$, 3 syringes $(12 \mathrm{ml})$ of KORTUC II were injected at each time point. This method was similar to that used in a previous phase I clinical trial started in 2017 by Nimalasena et al. [21]. Freehand transvaginal intratumor injection was performed under local anesthesia. We inserted an injection needle with the right hand and guided it to the tumor using left hand from inside the rectum.

ISBT was performed as a boost under general anesthesia, 18 days after the completion of EBRT. Our HDR-ISBT technique has been described in detail elsewhere [22]. In short, 13 flexible needle applicators (ProGuide sharp needle ${ }^{\circledR}$; Nucletron, an Elekta company, Elekta $\mathrm{AB}$, Stockholm, Sweden) were implanted transperineally using a freehand technique without template guidance under transrectal ultrasonography (TRUS). Subsequently, the applicators were cut short in order to allow the patient to sit and/or stand up. The KORTUC II application was then performed (Figure 2A), followed by CT imaging for anatomy-oriented HDR treatment planning (slice thickness $2 \mathrm{~mm}$, AquilionLB ${ }^{\circledR}$, Canon Medical Systems, Tochigi, Japan). Since catheter distribution was not deemed optimal, an additional applicator was implanted under CT guidance. KORTUC II was injected between applicators because the area between applicators showed a relatively low-dose irradiated area, which was similar to the basal dose points.

The final treatment plan was carried out using CT/ MRI co-registration for improved target and OARs de- tection [23] (Figure 2B). Povidone iodine gel was injected into the rectum to assist in visualization of the mucosal surface by a CT component. The treatment plan included measurement of gross tumor volume and OARs delineation with clinical target volume (CTV). Planning target volume (PTV) was generated from a CTV by adding a $10 \mathrm{~mm}$ cranial margin $[24,25]$.

Treatment plan evaluation was based on dose-volume histogram (DVH) analysis under consideration of the dose covering $90 \%\left[\mathrm{D}_{90}(\mathrm{CTV})\right], 98 \%\left[\mathrm{D}_{98}(\mathrm{CTV})\right]$, and $100 \%$ of the CTV $\left[\mathrm{D}_{100}(\mathrm{CTV})\right]$. Our dosimetric goal was $\mathrm{D}_{100}(\mathrm{CTV}) \geq$ planning aim dose (PAD), while allowing for $\mathrm{D}_{100}(\mathrm{CTV})<\mathrm{PAD}$ in case of excessive OARs doses, in which our dosimetric goal was $\mathrm{D}_{90}(\mathrm{CTV})$ or $\mathrm{D}_{98}$ (CTV) $>$ PAD. For DVH-based OARs evaluation, we considered the minimum dose received by the maximally irradiated $2 \mathrm{cc}\left(\mathrm{D}_{2 \mathrm{cc}}\right)$. The HDR-ISBT protocol consisted of $5 \mathrm{~Gy}$ fractional doses b.i.d. up to a total physical PAD of $25 \mathrm{~Gy}$ over 3 days, with an interfraction interval $>6 \mathrm{~h}$. After the final HDR treatment, the applicators were explanted. Considering an $\alpha / \beta=10 \mathrm{~Gy}$, the prescribed PAD generated a biological effective dose of $97.5 \mathrm{~Gy}$, with $10 \mathrm{~Gy}$ making an equivalent total dose in $2 \mathrm{~Gy}$ fractions $\left(\mathrm{EQD}_{2}\right)$ of $81.3 \mathrm{~Gy}$. In accordance with the DVH analysis, the volume to be irradiated with the prescribed dose as well as the volume covered by the $150 \%$ isodose amounted to $204.6 \mathrm{cc}$ and $87.7 \mathrm{cc}$, respectively. The CTV and PTV volumes were $89.7 \mathrm{cc}$ and $118.3 \mathrm{cc}$, respectively. The $\mathrm{D}_{90}(\mathrm{CTV}), \mathrm{D}_{98}(\mathrm{CTV})$, and $\mathrm{D}_{100}$ (CTV) were 6.0, 5.0, and 3.5 Gy per fraction, respectively. Similarly, $\mathrm{D}_{90}$ (PTV), $\mathrm{D}_{98}(\mathrm{PTV})$, and $\mathrm{D}_{100}$ (PTV) were 5.1, 3.8, and 2.8 Gy per fraction, respectively.

Dose limits for total $\mathrm{EQD}_{2}$ of OARs were $<90 \mathrm{~Gy}$ for the bladder, $<75$ Gy for the rectum, $<75$ Gy for the sigmoid colon, and $<75$ Gy for the small bowel (Table 1). This criterion was based on the EMBRACE II study [26]. Dose constraints of the sciatic nerve was not determined.

The $\mathrm{D}_{2 \mathrm{cc}}$ values were $2.1,4.1,3.2$, and 2.0 Gy per fraction for the bladder, rectum, sigmoid colon, and small bowel, respectively. With the addition of EBRT, the total $\mathrm{EQD}_{2}$ of $\mathrm{D}_{2 \mathrm{cc}}$ values were 60.7, 79.1, 69.8, and 60 Gy per
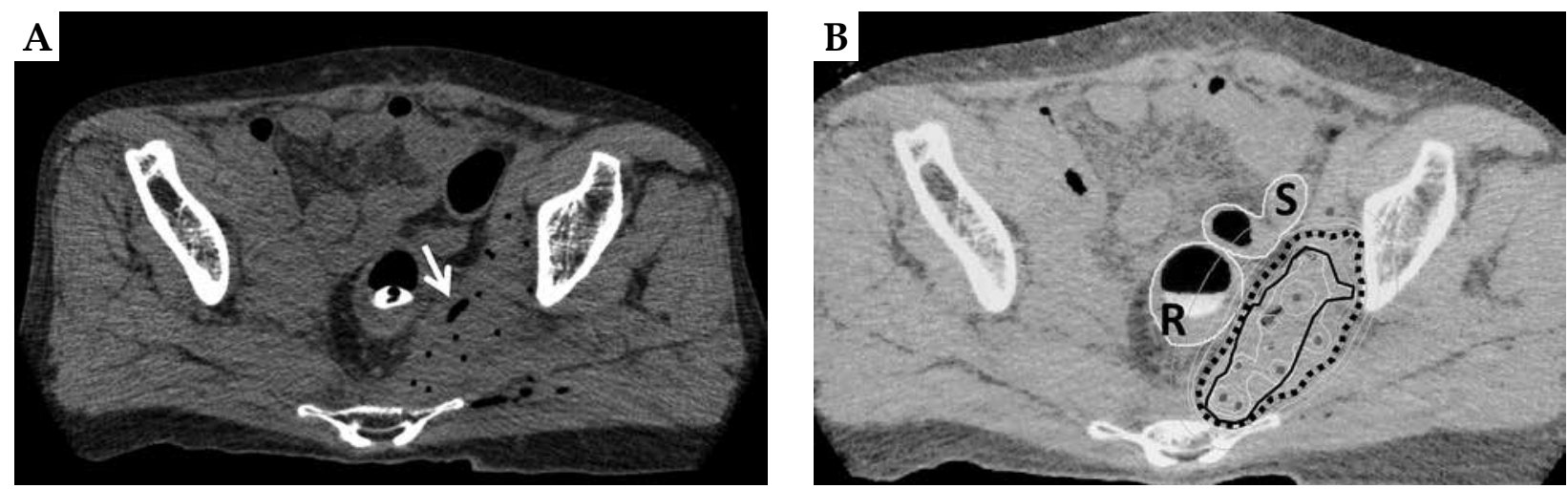

Fig. 2. A) Computed tomography of the same patient with 14 flexible needle applicators in situ. The white arrow indicates oxygen gas through the injection of sodium hyaluronate mixed with hydrogen peroxide into the tumor (the KORTUC II treatment). B) Isodose distribution of the interstitial brachytherapy. The $100 \%$-isodose line indicates the prescribed dose (5 Gy, black dotted line) covering the CTV (black solid line) without delivering excessive doses to the rectum (R) and the sigmoid colon (S). Povidone iodine gel was inserted into the rectum in order to visualize the mucosal surface clearly 
Table 1. The dose limits of organs at risk for treatment planning. $E Q D_{2}$ is calculated using $\alpha / \beta=3$. The total $\mathrm{EQD}_{2}$ include $50 \mathrm{~Gy} / 25$ fractions delivered by EBRT

\begin{tabular}{ccccc} 
& Bladder $\mathrm{D}_{2 c c}$ & Rectum $\mathrm{D}_{2 c c}$ & Sigmoid colon $\mathrm{D}_{2 c c}$ & Small bowel $\mathrm{D}_{2 c c}$ \\
\hline Dose limits & $<90 \mathrm{~Gy}$ & $<75 \mathrm{~Gy}$ & $<75 \mathrm{~Gy}$ & $<75$ Gy
\end{tabular}

fraction for the bladder, rectum, sigmoid colon, and small bowel, respectively.

The $\mathrm{D}_{0.1 \mathrm{cc}}$ value for the left sciatic nerve was at least 4.1 Gy. The total $\mathrm{EQD}_{2}$ of $\mathrm{D}_{0.1 \mathrm{cc}}$ value for the left sciatic nerve was at least 79.1 Gy. However, the left sciatic nerve could not be completely isolated because it was surrounded by the tumor lesion. Hence, the $\mathrm{D}_{0.1 \mathrm{cc}}$ values may be higher than the above-mentioned values.

All treatments were performed using an Iridium-192 ( $\left.{ }^{192} \mathrm{Ir}\right)$ HDR-afterloading system (MicroSelectron-HDR; Elekta AB, Stockholm, Sweden), with an apparent initial source activity of approximately $370 \mathrm{GBq}$.

The patient was followed up regularly and demonstrated very good clinical response. The follow-up MRI at 8 months after completion of treatment showed a complete radiological response according to RECIST criteria (Figure 3). The last follow-up CT at 32 months after ISBT, demonstrated enduring local control (LC) without newly diagnosed intrapelvic deposits. SCC tumor marker decreased to 0.5 .

Regarding toxicity, adverse events were scored according to the terminology criteria for adverse events version 4.0 (CTCAE v4), and no acute complications $\geq$ grade 3 were observed after HDR-ISBT combined with KORTUC II. In terms of late complications, the patient described left-sided sciatic neuralgia at 7 months after the treatment, which was scored as grade 2 , and still persisted at the time of writing. Synchronous grade 2 left lower limb lymphedema and urinary incontinence developed, showing no improvement during further follow-up. Thigh circumference was measured at the level of $10 \mathrm{~cm}$ below the femoral head, and the difference increased from $1.5 \mathrm{~cm}$ to $3.3 \mathrm{~cm}$ from pre-radiotherapy to 29 months after ISBT. Urinary incontinence was influenced by pyelonephritis; however, slight stress incontinence was also present at the time of writing this report. Repeated grade 3 pyelonephritis occurred at 11-, 24-, and 26-months post-treatment, and fully resolved after temporary intravenous antibiotic treatment.

\section{Discussion}

The management of recurrent uterine cervical cancer after definitive previous treatment, poses a challenge to the clinician. In fact, locoregional disease progression represents the most common cause of death in this gynecologic malignancy [27], with therapeutic options being limited and rarity of high-quality evidence. However, it should be emphasized that if locoregional disease is left untreated, the prognosis and quality of life are quite poor.

Although surgical resection constitutes the primary curative option when recurrent disease is resectable, but in reality, only a small group of patients is suited for this approach [28]. Few reports exist concerning the re- section of pelvic sidewall recurrent cancer [29,30]. Höckel reported on laterally extended endopelvic resection for pelvic wall recurrences [29]. He surgically treated 18 patients with or without post-operative radiotherapy, and a 3 -year survival rate was $40 \%$. However, this patients were carefully selected prior to surgery according to indications that patients presented with infra-iliac lesions, the tumor was $\leq 5 \mathrm{~cm}$, and had developed $>5$ months after a primary treatment.

Chemotherapy alone, with or without anti-vascular endothelial growth factor monoclonal antibody, showed a median survival of 13-17 months [31].

When irradiation is the only remaining salvages option, ISBT has demonstrated effectiveness in the management of recurrent uterine cervical cancer $[3,4,5,6,7,8,9,10$, $11,12,13,14,15,16,17]$. However, there are no well-defined recommendations for selecting patients for interventional radio-oncologic treatment, and each case is analyzed individually. For example, Jensen et al. treated 34 patients with locally advanced or recurrent gynecologic cancer using PDR-ISBT, and a 2-year survival rate was $63 \%$. PDR-ISBT combines the advantages of both LDR-ISBT (relatively lower dose-rate, which is theoretically safer than HDR-ISBT) and HDR-ISBT (no radiation exposure for medical staff) [15]. However, in Japan, the physician is obliged to accompany the patient during all irradiation sessions, which makes this procedure too difficult to accomplish and, as a result, PDR-ISBT is not used in Japan.

Some prognostic factors have been described for salvage radiotherapy with tumor size playing an important role $[6,8,11,13,14]$. Charra et al. examined the use of

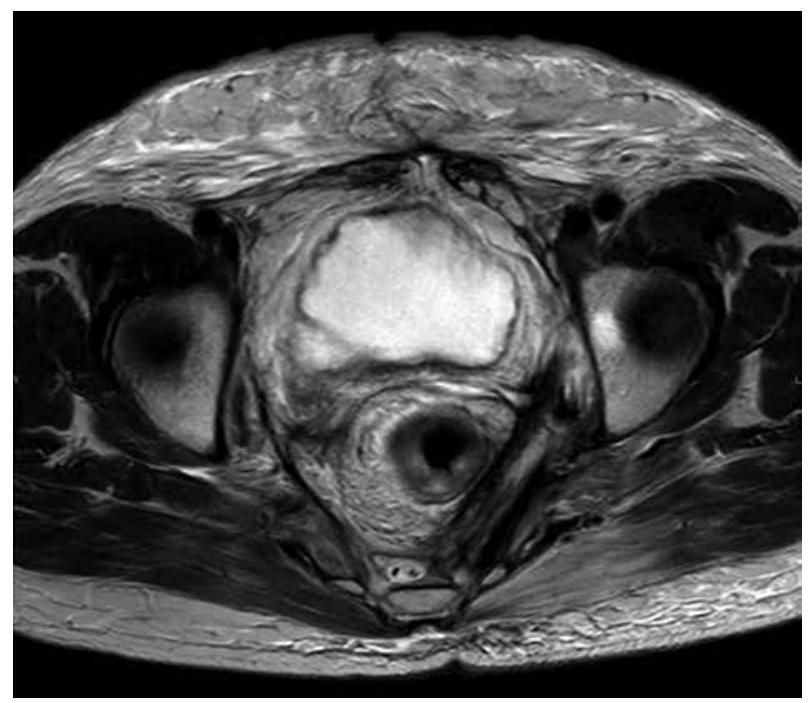

Fig. 3. Magnetic resonance imaging taken 8 months after interstitial brachytherapy as a part of follow-up. In the T2-weighted sequence, a long-term complete clinical remission can be verified 
LDR-ISBT in a treatment of vaginal recurrences of uterine cervical cancer, and showed that tumor diameter was a significant prognostic factor for a 5-year overall survival, which decreased from $63 \%$ to $37 \%$ if a recurrent lesion measured $\geq 4 \mathrm{~cm}$ [6]. Gupta et al. reported similar results on HDR-ISBT for locally advanced or recurrent gynecological malignancies, stressing tumor size as a significant prognostic factor with deterioration of a 3-year LC from $89 \%$ to $0 \%$ when a recurrent lesion volume was $\geq 100 \mathrm{cc}$ [8].

In addition to lesion size, tumor location seems also to be associated with clinical outcome $[3,4,7,11]$. Tan et al. reported on 100 patients treated for post-operative recurrent uterine cervical cancer using radical radiotherapy with or without chemotherapy, and found a 5-year survival rate of $42 \%$ for centrally located lesions vs. $15 \%$ for peripheral recurrences [4]. Likewise, Ijaz et al. reported on radiotherapy of pelvic recurrences after radical hysterectomy for cervical carcinoma. Their study encompassed 43 patients treated with or without chemotherapy, yielding a 5-year survival rate of $69 \%$ for central recurrence alone vs. $18 \%$ for recurrent tumors with sidewall extension [7].

In our case, the volumetrically calculated tumor volume was $89.7 \mathrm{cc}$ with deep extension into the pelvic sidewall. From this perspective, we considered KORTUC II a meaningful treatment option to restrict dose prescription through enhanced radiosensitivity $[19,20]$. Although our dose fraction schedule was slightly higher than those of other institutes $\left(\mathrm{EQD}_{2}\right.$ of 75.5-79.6) [32], the biological effects compared favorably with the recommended schedules in the ABS guidelines. Ogawa et al. reported the use of KORTUC II for unresectable or recurrent neoplasms, including sarcoma and malignant melanoma [20]. They treated 52 patients with a total of 53 lesions by EBRT. Their cohort included 31 patients with breast cancer and 8 with soft tissue sarcoma. The EBRT dose regimen was 49.5 Gy in 18 fractions for breast cancer and 54 Gy in 27 fractions for soft tissue sarcoma. The mixed complete response rate was $57 \%$ with a 2-year disease-free survival of $37 \%$, although the vast majority of cases consisted of large tumors and radioresistant histologic subtypes.

However, in cases with deep-seated large tumor like our patient, it is relatively difficult to inject KORTUC II homogeneously into the whole tumor area and, in some patients, the treatment may spread throughout the body causing damage to other organs. Additional care is required to ensure the safety of KORTUC II injections when treating patients with deep-seated large tumors, which require the use of a large number of long needles, as both the KORTUC II injection and needle applicator implantation may cause mechanical trauma, such as small bowel perforation [33]. The freehand injection technique used to administer KORTUC II during EBRT period must always be carried out in conjunction with CT/TRUS guidance as a standard procedure. We injected KORTUC II and implanted needle applicators with CT and TRUS guidance at the time of ISBT, as this constitutes a safer and more reproducible technique. Such image-guided implantation is also important in order to enable dose escalation to tumor while reducing dose to OARs. Furthermore, KORTUC II injection in a relatively lower dose irradiated areas, such as between applicators, offers a more effective method. We believe that further studies on the application of KORTUC II, focusing on overcoming difficulties associated with delivering the prescribed radiation doses to large deep-seated tumors without any cold spots, are necessary. Nimalasena et al. recently completed a phase I trial using EBRT with KORTUC II in locally advanced breast cancer patients [21]. Although, all tumors were $\geq 30 \mathrm{~mm}$, and 11 of the 12 patients maintained a partial or complete response (median follow-up, 12 months). Even though the KORTUC II procedure is easy for the treatment of superficial lesions, a safer and more stable technique remains to be found for large deep-seated tumors worldwide.

Based on the proven performance of KORTUC combined with radiotherapy, we offered KORTUC II treatment during EBRT and ISBT to our patient. After obtaining an informed consent, we carried out the procedure, which yielded improved radiosensitivity of hypoxic tumor cells that typically occurs during whole-pelvis EBRT, and ameliorated the radiation response in potential 'cold spot areas' caused by HDR-ISBT. The driving force behind this strategy was the provision of a curative treatment in a case of local pelvic recurrence of uterine cervical cancer with negative prognostic factors. Currently, 32 months post-treatment, the patient remains free of disease progression, with no further late severe adverse events. The toxicity profile is in line with the literature, in which gastrointestinal and genitourinary adverse events $\geq$ grade 3 are reported in up to $29 \%$ after radiotherapy for gynecological intrapelvic recurrences, respectively $[3,4,5$, $6,7,8,9,10,11,12,13,14,15,16,17]$.

\section{Acknowledgements}

This work was supported by JSPS KAKENHI (Grantin-Aid for Scientific Research C, with grant numbers JP17K10496, JP18K07639, JP17K10488, JP16K11534, and JP15K15461) as well as in part by the Japan Agency for Medical Research and Development (JP18ck0106305h002, JP19ck0106305h003).

We would like to thank Kenichiro Yamamura, Hiroki Okumura, RTT, radiation technologists, physicists, nursing staff, and other staff of the departments of Radiation Oncology, Radiology, Obstetrics and Gynecology, and Anesthesiology for their contribution for this work.

\section{Disclosure}

One of the authors (Ken Yoshida) assumes an advisory role of Chiyoda Technol ${ }^{\circledR}$ dealing with remote afterloader (microSelectron-HDR) in Japan. The other authors have no potential conflict of interest relevant to this manuscript to declare.

\section{References}

1. Miller B, Morris M, Rutledge F et al. Aborted exenterative procedures in recurrent cervical cancer. Gynecol Oncol 1993; 50: 94-99. 
2. Maneo A, Landoni F, Cormio G et al. Concurrent carboplatin/5-fluorouracil and radiotherapy for recurrent cervical carcinoma. Ann Oncol 1999; 10: 803-807.

3. Deutsch M, Parsons JA. Radiotherapy for carcinoma of the cervix recurrent after surgery. Cancer 1974; 34: 2051-2055.

4. Tan R, Chung CH, Liu MT et al. Radiotherapy for postoperative recurrent uterine cervical carcinoma. Acta Oncol 1991; 30: 353-356.

5. Corn BW, Lanciano RM, Rosenblum $\mathrm{N}$ et al. Improved treatment planning for the Syed-Neblett template using endorectal-coil magnetic resonance and intraoperative (laparotomy/ laparoscopy) guidance: a new integrated technique for hysterectomized women with vaginal tumors. Gynecol Oncol 1995; 56: 255-261.

6. Charra C, Roy P, Coquard R et al. Outcome of treatment of upper third vaginal recurrences of cervical and endometrial carcinomas with interstitial brachytherapy. Int J Radiat Oncol Biol Phys 1998; 40: 421-426.

7. Ijaz T, Eifel PJ, Burke T et al. Radiation therapy of pelvic recurrence after radical hysterectomy for cervical carcinoma. Gynecol Oncol 1998; 70: 241-246.

8. Gupta AK, Vicini FA, Frazier AJ et al. Iridium-192 transperineal interstitial brachytherapy for locally advanced or recurrent gynecological malignancies. Int J Radiat Oncol Biol Phys 1999; 43: 1055-1060.

9. Tewari K, Cappuccini F, Brewster WR et al. Interstitial brachytherapy for vaginal recurrences of endometrial carcinoma. Gynecol Oncol 1999; 74: 416-422.

10. Nag S, Yacoub S, Copeland LJ et al. Interstitial brachytherapy for salvage treatment of vaginal recurrences in previously unirradiated endometrial cancer patients. Int J Radiat Oncol Biol Phys 2002; 54: 1153-1159.

11. Weitmann HD, Knocke TH, Waldhäusl C et al. Ultrasound-guided interstitial brachytherapy in the treatment of advanced vaginal recurrences from cervical and endometrial carcinoma. Strahlenther Onkol 2006; 18: 86-95.

12. Viswanathan AN, Cormack R, Holloway CL et al. Magnetic resonance-guided interstitial therapy for vaginal recurrence of endometrial cancer. Int J Radiat Oncol Biol Phys 2006; 66 91-99.

13. Okazawa K, Yuasa Nakagawa K, Yoshimura R et al. Permanent interstitial re-irradiation with Au-198 seeds in patients with post-radiation locally recurrent uterine carcinoma J Radiat Res 2013; 54: 299-306.

14. Mabuchi S, Takahashi R, Isohashi $F$ et al. Reirradiation using high-dose-rate interstitial brachytherapy for locally recurrent cervical cancer. Int J Gynecol Cancer 2014; 24: 141-148.

15. Jensen PT, Roed H, Engelholm SA et al. Pulsed dose rate (PDR) brachytherapy as salvage treatment of locally advanced or recurrent gynecologic cancer. Int J Radiat Oncol Biol Phys 1998; 42: 1041-1047.

16. Kotsuma T, Yoshida K, Yamazaki H et al. Preliminary results of magnetic resonance imaging-aided high-dose-rate interstitial brachytherapy for recurrent uterine carcinoma after curative surgery. J Radiat Res 2011; 52: 329-334.

17. Yoshida K, Yamazaki H, Kotsuma T et al. Treatment results of image-guided high-dose-rate interstitial brachytherapy for pelvic recurrence of uterine cancer. Brachytherapy 2015; 14: $440-448$.

18. Ogawa Y, Takahashi T, Kobayashi T et al. Apoptotic-resistance of the human osteosarcoma cell line HS-Os-1 to irradiation is converted to apoptotic-susceptibility by hydrogen peroxide: a potent role of hydrogen peroxide as a new radiosensitizer. Int J Mol Med 2003; 12: 845-850.

19. Ogawa $Y$, Kubota K, Ue H et al. Phase I study of a new radiosensitizer containing hydrogen peroxide and sodium hyaluronate for topical tumor injection: A new enzyme-targeting radiosensitization treatment, Kochi Oxydol-Radiation Therapy for Unresectable Carcinomas, Type II (KORTUC II). Int J Oncol 2009; 34: 609-618.

20. Ogawa Y, Kubota K, Ue H et al. Safety and effectiveness of a new enzyme-targeting radiosensitization treatment (KORTUC II) for intratumoral injection for low-LET radioresistant tumors. Int J Oncol 2011; 39: 553-560.

21. Nimalasena $S$, Gothard L, Anbalagan S et al. Intratumoural hydrogen peroxide with radiotherapy in locally advanced breast cancer: results from a phase I clinical trial. Int J Radiat Oncol Biol Phys 2020; 108: 1019-1029.

22. Yoshida K, Nose T, Shiomi H et al. New ambulatory implant technique of high-dose-rate interstitial brachytherapy for prostate cancer. Radiat Med 2006; 24: 595-599.

23. Yoshida K, Nose T, Koizumi M et al. The usefulness of metal markers for CTV-based dose prescription in high-dose-rate interstitial brachytherapy. J Jpn Soc Ther Radiol Oncol 2002; 13: 253-260.

24. Yoshida K, Ueda M, Takenaka T et al. Daily CT measurement of needle applicator displacement during multifractionated high-dose-rate interstitial brachytherapy for postoperative recurrent uterine cancer. J Radiat Res 2012; 53: 295-300.

25. Mikami M, Yoshida K, Takenaka T et al. Daily computed tomography measurement of needle applicator displacement during high-dose-rate interstitial brachytherapy for previously untreated uterine cervical cancer. Brachytherapy 2011; 10: 318-324.

26. Pötter R, Tanderup K, Kirisits $C$ et al. The EMBRACE II study: The outcome and prospect of two decades of evolution within the GEC-ESTRO GYN working group and the EMBRACE studies. Clin Transl Radiat Oncol 2018; 9: 48-60.

27. Lucraft HH. Radiotherapy following primary surgery for carcinoma of uterine cervix. Clin Radiol 1981; 32: 347-353.

28. National Cancer Institute. Cervical cancer treatment (PDQ®) - Health professional version. https://www.cancer.gov/ types/cervical/hp/cervical-treatment-pdq\#_147 (accessed 10 May 2020).

29. Höckel M. Laterally extended endopelvic resection: surgical treatment of infrailiac pelvic wall recurrences of gynecologic malignancies. Am J Obstet Gynecol 1999; 180: 306-312.

30. Kanao H, Aoki Y, Kato K et al. Total laparoscopic resection surgery for a cervical carcinoma that recurred in the pelvic sidewall after radical hysterectomy and adjuvant concurrent chemoradiotherapy. Minim Invasive Gynecol 2017; 24 899-900.

31. Tewari KS, Sill MW, Long HJ 3rd et al. Improved survival with bevacizumab in advanced cervical cancer. $N$ Engl J Med 2014; 370: 734-743.

32. Albuquerque $\mathrm{K}$, Hrycushko BA, Harkenrider MM et al. Compendium of fractionation choices for gynecologic HDR brachytherapy - an American Brachytherapy Society Task Group Report. Brachytherapy 2019; 18: 429-436.

33. Masui K, Yamazaki H, Suzuki G et al. Small bowel perforation caused by applicator implantation in high-dose-rate interstitial brachytherapy for recurrent pelvic tumor: a case report. J Contemp Brachytherapy 2020; 12: 188-192. 